\title{
Implementation of a Systematic Literature Search Strategy in a National Breast Cancer Guideline: The Relevance of Systemic Therapy in Lymph Node Recurrent Disease
}

\author{
Tanja N. Stüber ${ }^{\mathrm{a}}$ Stephanie Stangl $^{\mathrm{b}}$ Christian Jackisch $^{\mathrm{c}}$ Wolfgang Janni $^{\mathrm{d}}$ Sara Y. Brucker $^{\mathrm{e}}$ \\ Andreas Hartkopf ${ }^{e}$ Bernd Gerber ${ }^{f}$ Wilfried Budach ${ }^{g}$ Michael P. Lux ${ }^{\mathrm{h}} \quad$ Achim Wöckel $^{\mathrm{a}}$

\footnotetext{
a Department of Gynecology and Obstetrics, University of Würzburg Medical School, Würzburg, Germany;

b Institute of Clinical Epidemiology and Biometry, University of Würzburg, Würzburg, Germany;

${ }^{c}$ Department of Obstetrics and Gynecology, Sana hospital Offenbach, Offenbach, Germany;

${ }^{\mathrm{d}}$ Department of Gynecology and Obstetrics, University UIm, UIm, Germany;

e Department of Gynecology and Obstetrics, University of Tübingen, Tübingen, Germany;

f Department of Gynecology and Obstetrics, University of Rostock, Rostock, Germany;

gDepartment of Radiotherapy and Radiation Oncology, University of Düsseldorf, Düsseldorf, Germany;

${ }^{h}$ Department of Gynecology and Obstetrics, University of Erlangen, Erlangen, Germany
}

\section{Keywords}

Breast cancer - Chemotherapy - Disease management .

Guideline - Lymph node recurrent disease .

Systematic literature search

\section{Summary}

Purpose:Our aim was to implement a structured literature search using PICO (patient/intervention/comparison/ outcome) questions and a standardized consensus method for the German Guideline 'Detection, diagnostics, therapy and follow-up of Breast Cancer' using, as an example, the significance of systemic therapy in lymph node recurrent disease (LNRD). Methods: We defined specified PICO questions according to the clinical significance of a recommendation for systemic therapies in locoregional LNRD. A methodologist performed a systematic literature search including randomized controlled trials, systematic reviews and observational studies (2007-2016). In a consensus conference, the level of evidence and consensus was determined and the clinical recommendation was adopted. Results: In total 143 publications were identified according to the search strategy including 14 duplicates, which were excluded. 4 publications were included based on experts' choice. The team excluded 119 publications, leaving 14 that were then screened by a full text search. Finally, 1 publication was found to be of methodologically and clinically reliable content. The conclusion of this publication in favor of systemic therapy for LNRD received strong consent from the consensus conference. Conclusions: The literature search strategy using PICO questions helped to achieve a fast and standardized selection of publications. The recommendation concerning systemic treatment of LNRD was first implemented in the update of the German Breast Cancer guideline.

(c) 2018 S. Karger GmbH, Freiburg

\section{Introduction}

Breast cancer continues to be the most frequent cancer among women. In Germany for example, in 2013, 72,000 cases were reported and almost 18,000 patients died [1]. The treatment of breast cancer is an interdisciplinary approach involving among others gynecologists, senologists, oncologists and radiologists. Clinical guidelines can improve decision making concerning appropriate care. The guidance reflects the current state of scientific research and expert knowledge assessed by a systematic approach.

In Germany, the Guideline Program in Oncology (Leitlinienprogramm Onkologie) fosters the development, implementation

\section{KARGER}

() 2018 S. Karger GmbH, Freiburg 
and evaluation of evidence-based clinical oncological guidelines. The program was initiated by the Association of Scientific Medical Societies (AWMF), the German Cancer Association (DKG) and the German Cancer Aid (DKH) in 2008. The guideline 'Detection, Diagnosis, Therapy, and Follow-Up of Breast Cancer' is classified as level S3 according to the AWMF Guidance Manual and Rules [2]. This level is the highest (out of 3: S1, S2 (S2k, S2e), S3) and represents an evidence- and consensus-based approach: a representative committee, systematic review and accrual of evidence and a structured consensus development. The German Society of Gynecology and Obstetrics (DGGG) and the German Cancer Society (DKG) update the version every 5 years. The development or updating of guidelines is a work-, time-, and cost-intensive process, which requires a sensible utilization of the available resources. Most of the statements and recommendations of the German breast cancer guideline were revised based on recommendations of existing guidelines, which were systematically searched and reviewed with the AGREE II instrument [3]. Guidelines were considered qualitatively eligible if domain 3 (Rigor of development) was assessed with $>50 \%$. Moreover, we carried out a systematic search and accrual of evidence from systematic reviews, clinical trials or observational studies for 17 recommendations, 1 of which focused on systemic therapy of recurrent breast cancer.

The recurrence risk after primary breast cancer varies depending on tumor size, tumor biology and tumor treatment. In 10-20\% of cases, recurrence occurs in isolated locoregional sites [4]. Besides local surgery to remove all tumorous lesions and radiotherapy, there is still need for recommendations concerning systemic therapy in lymph node recurrent disease (LNRD). In the following, we illustrate the applied methodology for identification, selection, appraisal of evidence from systematic reviews and individual studies and the agreement on recommendations for the current S3 guideline for breast cancer.

\section{Methods}

\section{Systematic Search and Selection}

The methodology to update the evidence- and consensus-based S3 guideline for breast cancer is in accordance with the AWMF Manual Guidance and Rules as mentioned above. The DGGG and DKG designated a co-ordination and project team to steer the process, and a multi-professional expert panel was set up.

We defined research questions based on PICO (patient/ population, intervention, comparison (treatment), outcome) criteria according to the clinical significance of a recommendation for systemic therapies in LNRD [5]. The key question comprised whether the treatment of breast cancer patients (initial diagnosis $\mathrm{pN} 0-\mathrm{pN}^{+}$) with ipsilateral, supraclavicular and even contralateral lymph node disease with systemic therapies (chemotherapy/endocrine therapy) is superior to operation and/or radiation with respect to progression-free survival (PFS), overall survival (OS) and quality of life (QoL). The population was defined as breast cancer patients (female/male) with recurrent lympho-regional disease or contralateral recurrent disease independent of prior therapies without further distant metastases. Interventions were characterized as endocrine therapy in the case of endocrine responsive recurrent disease, chemotherapy or goal-directed therapies according to the tumor profile. Additionally, surgical resections were included if technically feasible. Outcomes were defined as locoregional recurrence, PFS, OS, QoL and acute and late-onset toxicities. The search was restricted to systematic reviews and meta-analyses, randomized controlled trials (RCTs) and observational studies. Search filters from the Scottish Intercollegiate Guideline Network (SIGN) were used to restrict the results to systematic reviews and meta-analysis or RCTs. Only publications in the English or German language, published between 2007 and 'current' (i.e. date of search carried out (29 June 2016)) were eligible. Inclusion and exclusion criteria were derived from the PICO criteria (table 1).

The search was carried out on 29 June 2016 in the electronic databases Medline (via Ovid), Cochrane Database of Systematic Reviews (CDSR), Cochrane Centre Register of Controlled Trials (CENTRAL) (via Cochrane Library), Database of Abstracts of Reviews of Effects (DARE) (via Pub Med Health) (table 2) and clinical trial registries (clinicaltrial.gov and EU clinical trials registry). The working group searched for additional publications, which were included into the literature search process if marked as an expert contribution.

Table 1. Inclusion and exclusion criteria

\begin{tabular}{|c|c|}
\hline \multicolumn{2}{|l|}{ Inclusion criteria } \\
\hline E1: target group/topic & $\begin{array}{l}\text { population/topic as described in PICO criteria/ } \\
\text { key question }\end{array}$ \\
\hline E2: publication type & $\begin{array}{l}\text { systematic review and meta-analysis, RCT, } \\
\text { observational studies }\end{array}$ \\
\hline E3: search period & 2007-current (29.06.2016) \\
\hline E4: languages & German or English \\
\hline E5: Intervention & intervention as described in PICO criteria \\
\hline E6: Compare & control treatment as described in PICO criteria \\
\hline \multicolumn{2}{|l|}{ Exclusion criteria } \\
\hline A1 & $\begin{array}{l}\text { different target group or topic (i.e. different } \\
\text { disease) }\end{array}$ \\
\hline $\mathrm{A} 2$ & different intervention \\
\hline A3 & different control treatment \\
\hline A4 & different outcome \\
\hline A5 & $\begin{array}{l}\text { different publication type (i.e. narrative review, } \\
\text { editorial, letter, case report etc.) }\end{array}$ \\
\hline A6 & $\begin{array}{l}\text { double publication or more up-to-date } \\
\text { publication is available of this study }\end{array}$ \\
\hline
\end{tabular}

$\mathrm{PICO}=$ patient $/$ intervention/comparison/outcome, $\mathrm{RCT}=$ randomized controlled trial.

Table 2. Search strategy for RCTs

\begin{tabular}{|c|c|c|}
\hline \# & $\begin{array}{l}\text { Ovid MEDLINE(R) } 1946 \text { - June week } 32016 \\
\text { searches }\end{array}$ & Results, $\mathrm{n}$ \\
\hline 1 & $\begin{array}{l}\text { breast neoplasms/ or carcinoma, ductal, breast/ } \\
\text { or breast neoplasms, male/ or inflammatory } \\
\text { breast neoplasms/ }\end{array}$ & 243,771 \\
\hline 2 & $\begin{array}{l}\text { breast/ or mammary glands, human/ or nipples/ } \\
\text { or breast diseases/ }\end{array}$ & 47,854 \\
\hline 3 & neoplasms/ or adenocarcinoma/ or carcinoma/ & 566,366 \\
\hline 4 & 2 and 3 & 4,658 \\
\hline 5 & $\begin{array}{l}\text { (brca or (breast adj4 (adenocarcinoma* or } \\
\text { cancer }^{*} \text { or carcinoma* or metasta* } \text { or neoplasm }^{*} \\
\text { or tumo?r))).ti,ab,kw. }\end{array}$ & 230,787 \\
\hline 6 & 1 or 4 or 5 & 292,026 \\
\hline 7 & $\begin{array}{l}\text { neoplasms, second primary/ or neoplasm } \\
\text { recurrence, local/ or clavicle/ or recurrence/ or } \\
\text { axilla/ or lymph nodes/ or lymphatic metastasis/ }\end{array}$ & 398,574 \\
\hline
\end{tabular}

Table 2. Continued on next page 
Table 2. Continued

\begin{tabular}{|c|c|c|}
\hline \# & $\begin{array}{l}\text { Ovid MEDLINE(R) } 1946 \text { - June week } 32016 \\
\text { searches }\end{array}$ & Results, $\mathrm{n}$ \\
\hline 8 & $\begin{array}{l}\text { ((ipsilateral or contralateral or supraclavicular } \\
\text { or internal mamma*) adj3 (lymph* or lymph } \\
\left.\text { nod }^{*} \text { or metasta }{ }^{\star}\right) \text {.ti,ab,kw. }\end{array}$ & 3,035 \\
\hline 9 & 7 and 8 & 1,893 \\
\hline 10 & 6 and 9 & 755 \\
\hline 11 & limit 10 to $y r=" 2007$-Current" & 243 \\
\hline 12 & limit 11 to English & 221 \\
\hline 13 & limit 11 to German & 1 \\
\hline 14 & 12 or 13 & 222 \\
\hline 15 & meta-analysis as topic/ & 15,079 \\
\hline 16 & meta analy\$.tw. & 77,761 \\
\hline 17 & metaanaly\$.tw. & 1,478 \\
\hline 18 & meta-analysis/ & 67,381 \\
\hline 19 & (systematic adj (review $\$ 1$ or overview $\$ 1$ )).tw. & 66,759 \\
\hline 20 & exp review literature as topic/ & 8,751 \\
\hline 21 & or/15-20 & 147,447 \\
\hline 22 & cochrane.ab. & 37,164 \\
\hline 23 & embase.ab. & 37,303 \\
\hline 24 & (psychlit or psyclit).ab. & 860 \\
\hline 25 & (psychinfo or psycinfo).ab. & 8,847 \\
\hline 26 & (cinahl or cinhal).ab. & 12,363 \\
\hline 27 & science citation index.ab. & 2,181 \\
\hline 28 & bids.ab. & 365 \\
\hline 29 & cancerlit.ab. & 583 \\
\hline 30 & or/22-29 & 58,270 \\
\hline 31 & reference list\$.ab. & 11,064 \\
\hline 32 & bibliograph\$.ab. & 12,096 \\
\hline 33 & hand-search\$.ab. & 4,248 \\
\hline 34 & relevant journals.ab. & 810 \\
\hline 35 & manual search\$.ab. & 2,587 \\
\hline 36 & or/31-35 & 27,613 \\
\hline 37 & selection criteria.ab. & 21,706 \\
\hline 38 & data extraction.ab. & 10,978 \\
\hline 39 & 37 or 38 & 30,920 \\
\hline 40 & review/ & $2,069,843$ \\
\hline 41 & 39 and 40 & 22,094 \\
\hline 42 & comment/ & 632,631 \\
\hline 43 & letter/ & 891,703 \\
\hline 44 & editorial/ & 383,062 \\
\hline 45 & animal/ & $5,900,585$ \\
\hline 46 & human/ & $16,091,919$ \\
\hline 47 & 45 not (45 and 46$)$ & $4,233,089$ \\
\hline 48 & or/ $42-44,47$ & $5,584,098$ \\
\hline 49 & 21 or 30 or 36 or 41 & 176,114 \\
\hline 50 & 49 not 48 & 165,204 \\
\hline 51 & 14 and 50 & 11 \\
\hline
\end{tabular}

a Strategy was adapted according to publication type and electronic database). This table demonstrates the search filters for the evaluation of the PICO question. $\mathrm{RCT}=$ randomized controlled trial, $\mathrm{PICO}=$ patient/intervention/comparison/ outcome, Adj4 = adjacency searching helps to find the phrase within 4 words, $\mathrm{Ti}=$ term searched for in the title of the article, $\mathrm{Ab}=$ term searched in the $\mathrm{ab}$ stract of the article, Tw = textword index includes title and abstract, $\mathrm{Kw}=$ term searched in the keywords, ? = used to replace zero or one character, ${ }^{*}=$ search anything with the stem of the search term, $\$=$ alternative to indicate truncation, Or $=$ to combine specific search lines, And = to combine specific search lines, / = indicates a subject heading.

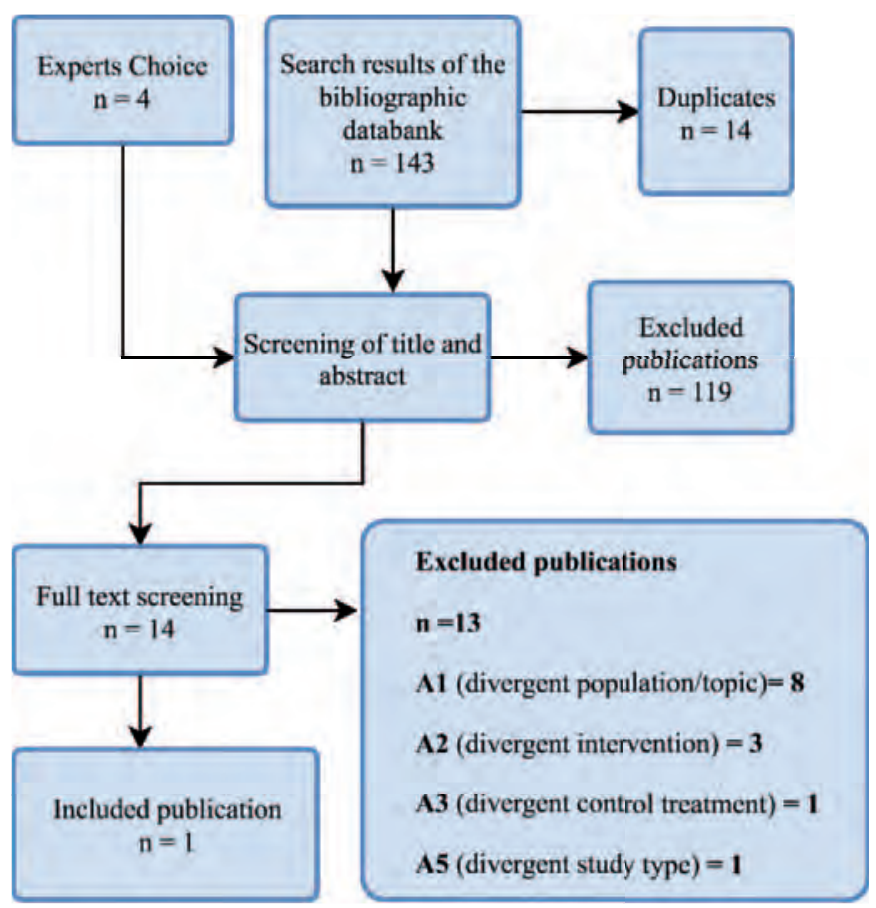

Fig. 1. Flow chart demonstrates the selection process of the literature search.

\section{Data Extraction and Critical Appraisal}

We downloaded the references of the search hits to Endnote reference manager. After removal of duplicates, we extracted information from the remaining references (i.e. author, year, journal, title, abstract, DOI number) to an Excel sheet and sent it to 2 independent raters for screening. The independent raters ( 2 senological gynecologists) were asked to assess the abstracts or titles of each publication for relevance according to the PICO criteria. The completed Excel sheets were sent back to the methodologist. Disagreements amongst raters were resolved by discussion or consultation with a third person. For the remaining publications, the full-text was obtained and screened for eligibility by 2 raters. Disagreement on relevance of the study according to the inclusion criteria was again resolved by discussion or by a third person. The final set of eligible studies was critically appraised using the SIGN checklist (Version March 2004) by 2 independent methodologists, and a level of evidence (LoE) (using the system of the Oxford Centre for evidence-based medicine Version 9) was assigned. If there were methodological constraints, the LoE was downgraded. Relevant data of the publications were extracted by a methodologist and presented in an evidence table to the working group (fig. 1).

\section{Guideline Recommendation and Consensus Conference}

In a first step, based on the study results, a recommendation draft for the consensus conference was formulated by the members of the locoregional recurrence working group on the S3 guideline. The working group discussed the draft during a phone conference moderated by a methodologist of the Guideline Program in Oncology. Finally, the strength of recommendation for the clinical recommendation was assessed in the final consensus conference of all members of the S3 guideline for breast cancer. The strength of recommendation was determined in relation to the level of evidence of the study by the whole guideline committee. The final clinical recommendation and the underlying level of evidence and strength of recommendation was then acquiesced by elective representatives of participating medical societies in a nominal group process moderated by a methodologist of the AWMF. All guideline members had to disclose their conflicts of interests to participate in the S3 guideline working process. In case of a relevant conflict of interest of elective members, they were excluded from the respective vote. The vote was carried out anony- 
mously using a TED-transmitter system. The results of all votes were classified according to the percentage consensus into: strong consensus, >95\% approval; consensus, > 75-95\% approval; majority consensus, > 50-75\%; and disagreement, $<50 \%$ approval

\section{Results}

According to our search strategy we retrieved 143 publications from the databases and clinical trial registries. After removing 14 duplicates and adding 4 expert contributions, 133 publications were screened on an abstract or title level by independent raters using predefined exclusion criteria. 119 publications were labeled irrelevant according to the PICO criteria. The full texts of the remaining 14 publications were retrieved and screened by 2 independent experts. 9 of these were excluded since they did not address the target group. Wang et al. [6] performed a retrospective analysis in a Chinese cohort of patients with contralateral axillary lymph node metastasis examined between 1999 and 2008. They showed that PFS was increased in patients who were treated with radiotherapy. Regarding the manuscript, only 25 patients with recurrent disease were analyzed retrospectively and the PICO question was not addressed since a comparison of treatment options was not possible. Another publication by Chen et al. [7]. described the frequency and risk factors of internal mammary lymph node (ILMN) recurrence. The group stated that the hormone receptor status is a prognostic factor for OS after ILMN recurrence but also did not answer our PICO question The work of Fulga et al. [8] received no consideration since they focused on describing the pathological characteristics of locoregional recurrence rather than comparison of the influence of different treatments on the outcome. Both Lanitis et al. [9] and Chang et al. [10] analyzed axillary metastatic disease or synchronous ipsilateral supraclavicular lymph node metastases as primary diagnosis rather than recurrence after the primary diagnosis of breast cancer. Ohsumi et al. [11] described the detection of ipsilateral regional lymph node recurrence using positron emission tomography-computed tomography. Moossdorff et al. [12] reviewed the literature to determine the prognosis after contralateral lymph node recurrence in breast cancer. They stated that locoregional and systemic treatment led to incomparable outcome with distant metastases and thus should be regarded as a regional event. Van Wely et al. [13] also did not meet the PICO question since they published a mini review showing the association between lower rates of local recurrence and external beam radiation after negative sentinel lymph node detection. Kaur et al. [14] reported that remapping of lymph nodes after prior completion of axillary lymph node dissection is feasible and should be considered regardless of the number of initially removed lymph nodes. Although these results are interesting for the management of recurrent disease, they still do not answer our question concerning a comparison of the treatment options in LNRD.

Three further publications focused on interventions other than those defined by the PICO question. An intriguing study published in 2010 by Fan et al. [15] compared metachronous and synchronous manifestations of recurrent disease, but did not systematically analyze differences between systemic treatment and regional treatment. Braunstein et al. [16] evaluated the influence of tumor subtype on the outcome of locoregional recurrent breast cancer. In this study $90 \%$ of patients with recurrent disease received surgical treatment, $11 \%$ radiotherapy, $37 \%$ chemotherapy and $28 \%$ endocrine therapy. Patients with recurrent disease and luminal A-type tumors were associated with better disease-free survival (DFS) and OS than those with triple-negative tumors. In the univariate analysis they found that surgical intervention improved DFS and interestingly the application of chemotherapy led to worse DFS, while radiotherapy and endocrine therapy did not influence the outcome. In 2011, Pedersen et al. [17] also examined the influences of different salvage therapies on the outcome in recurrent disease: $19 \%$ of the patients were operated to an at least macroscopic tumor-free status, $33 \%$ received radiotherapy, $40 \%$ endocrine therapy and $45 \%$ any type of systemic chemotherapy. In summary, $26 \%$ of all patients had combined regional and systemic treatment. Another consensus classification report evaluated publications describing various methodology and was thus excluded [18].

Finally, 1 publication was eligible. The open-label, randomized trial examined chemotherapy for isolated locoregional recurrence of breast cancer (CALOR) and was published in 2014 [19]. 162 patients with isolated locoregional recurrences after unilateral breast cancer and after primary treatment with mastectomy or lumpectomy were included. The median age was 56 years. Most of the patients were diagnosed with recurrent disease of the breast $(\mathrm{n}=89$; $54.9 \%)$ and the chest wall or scar $(\mathrm{n}=53 ; 32.7 \%)$, while 20 patients (12.3\%) had LNRD. Absence of metastatic disease, other malignancies prior to breast cancer and macroscopic clear margins after surgery for recurrent disease were inclusion criteria. Radiotherapy was recommended for all patients, while endocrine therapy was recommended for all hormone receptor (HR)-positive patients. The randomized allocation of systemic chemotherapy resulted in 2 groups (chemotherapy and no chemotherapy). The type of chemotherapy was administered according to physician's choice. DFS was set as primary endpoint, while secondary endpoint was OS. The median follow-up comprised 4.9 years. 80 patients (49.4\%) received chemotherapy and $94 \%$ of patients with HR-positive breast cancer received endocrine therapy. As 1 of the major findings, Aebi et al. [19] showed that the application of chemotherapy led to a significantly improved DFS (69\% vs. $57 \%$; hazard ratio 0.59 ), a reduction of distant and local failures and significantly improved OS (88\% vs. $75 \%$; hazard ratio 0.41 ). Due to methodological constraints, the methodologists proposed downgrading the level of evidence from $1 \mathrm{~b}$ to $2 \mathrm{a}$ according to the Oxford Centre for evidence-based medicine Version 9. These constraints included no blinding, broad 95\% confidence intervals, and no information about how many patients dropped out.

The team of gynecologists then prepared the clinical recommendation based on the literature assessment, which read: 'Systemic therapy after R0 resection of recurrent disease has to be recommended in order to achieve longer disease-free survival as well as overall survival'. There was strong consent for the recommendation among the voting participants of the consensus conference [20]. 


\section{Discussion}

The evidence-based methods using a predefined question (i.e. PICO criteria), a systematic search of the literature and an appraisal of the identified publications are a necessary tool to guarantee high quality, transparency and acceptance of the recommendations of the S3 guideline for breast cancer. In this work, we demonstrate a well-structured search strategy to gain evidence for clinical guideline recommendations using as an example the systemic therapy in lymph node recurrent breast cancer. The search strategy helped to filter relevant publications that were precisely screened concerning their medical contents. It helped us to discuss the current literature and to draft a clinical recommendation.

After the exclusion of unsuitable publications, only 1 study was considered as being of sufficient value to be the source of a clinical recommendation. The trial of Aebi et al. [19] perfectly matched our PICO question; however, there were methodological constraints. Those included the lack of HER2 testing, a short follow-up period, missing information on the drop-out rate, a broad $95 \%$ confidence interval and a study enrolment that did not reach the power of $80 \%$. Additionally, our central interest focused on the subgroup of patients who develop LNRD. In the CALOR trial, only 20 patients were diagnosed with LNRD, while most of the patients $(\mathrm{n}=142$ ) were diagnosed with chest wall, scar or breast recurrent disease. These concerns led to a downgrading of the level of evidence from $1 \mathrm{~b}$ to $2 \mathrm{a}$. Moreover, efficacy of chemotherapeutic treatment was predominant in cases of HR-negative tumors. In cases of HR-positive tumors, benefit could not be excluded and remains unclear based on the current data. In the retrospective study of Pedersen et al. [17] combined locoregional and systemic therapy were associated with longer PFS and OS compared to systemic or locoregional therapy alone. Even though the data were not randomized and chemotherapeutic regimens were not up to date, the results were in line with the CALOR trial. In contrast to those results, Braunstein et al. [16] showed that the application of chemotherapy led to worse outcome, while radiotherapy and endocrine therapy had no influence on the outcome. The authors suggested that the underpowered design on the one hand and the mainly HRpositive patients in their collective on the other hand led to this divergence of results. There is no doubt that histopathological examination using biopsies plays a crucial role for the management of recurrent disease and should be considered first before a therapeutic strategy is set up. Despite the lack of more methodically valua- ble publications, we were able to draft a recommendation that should be considered in systemic therapy in cases with recurrent breast cancer.

This recommendation is in line with the European Society of Medical Oncology (ESMO). EMSO stated in their third updated consensus guideline in February 2017 that, in the absence of distant metastases, the use of systemic therapy (chemotherapy, endocrine therapy and/ or anti-HER2-directed therapy) should be considered in addition to local therapy (surgery and/or radiation therapy). In comparison to our recommendation, the evidence level is 1b. The ESMO guideline, however, was not considered since the methodological evaluation of domain 3 according to the AGREE II instrument was below 50\%. Since 2010, the American Society of Oncology (ASCO) uses a formal consensus methodology, including quality appraisal, to rate the strength of evidence and the strength of recommendation [21]. In these aspects, the strategy of ASCO and AWMF are quite similar. So far, no ASCO guidelines have been published that give their view on systemic treatment of lymph node recurrent breast cancer.

There are several benefits, but also limitations, of the presented search strategy. Methodical constraints might relate to the time limitation of 16 years (2000-2016) of the inspected publications. Moreover, only publications in English and German were included; however, it might be reasonably assumed that most of the relevant methodical and high-valued publications are in English. A benefit of the strategy is that it represents a quick search especially tailored to filter all the relevant publications to answer the clinical question. Furthermore, inclusion and exclusion criteria were predefined before the search was carried out. Another strength is the gradually performed screening of the literature by senological gynecologists and the standardized consensus conference to guarantee a high standard of medical and methodical content.

In summary, we believe that the presented search strategy and methodology is important and should be considered when clinical guidelines are set up. Further investigations are required to substantiate the clinical recommendation made.

\section{Disclosure Statement}

Informed consent was not necessary. The study was supported by the University hospital of Wuerzburg. T.S. received honoraria by Roche Pharma and a research grant by Else-Kroener-Fresenius. The other authors declare that they do not have any conflicts of interests.

\section{References}

1 Robert-Koch-Institute, R., Bericht zum Krebsgeschehen in Deutschland 2016. 2016

2 Muche-Borowski C, Selbmann HK, Nothacker M, et al.: AWMF Guidance Manual and Rules for Guideline Development. English version, 1st ed. 2012.

- 3 Brouwers MC, Kho ME, Browman GP, et al., AGREE II: Advancing guideline development, reporting and evaluation in health care. CMAJ 2010;182:E839-842.
4 Gerber B, Freund M, Reimer T, Recurrent breast cancer: Treatment strategies for maintaining and prolonging good quality of life. Dtsch Arztebl Int 2010;107: 85-91.

5 Huang X, Lin J, Demner-Fushman D: Evaluation of PICO as a knowledge representation for clinical questions. AMIA Annu Symp Proc 2006:359-363.

6 Wang W, Yuan P, Wang J, et al.: Management of contralateral axillary lymph node metastasis from breast cancer: a clinical dilemma. Tumori 2014;100:600-604.
7 Chen L, Gu Y, Leaw S, et al.: Internal mammary lymph node recurrence: Rare but characteristic metastasis site in breast cancer. BMC Cancer 2010;10:479.

8 Fulga V, Rudico L, Balica AR, et al.: Invasive ductal carcinoma of no special type and its corresponding lymph node metastasis: Do they have the same immunophenotypic profile? Pol J Pathol 2015;66:30-37.

9 Lanitis S, Behranwala KA, Al-Mufti R, Hadjiminas D: Axillary metastatic disease as presentation of occult or contralateral breast cancer. Breast 2009;18:225-227. 
10 Chang XZ, Yin J, Sun J, et al.: A retrospective study of different local treatments in breast cancer patients with synchronous ipsilateral supraclavicular lymph node metastasis. J Cancer Res Ther 2013;9(Suppl):S158-161.

11 Ohsumi S, Inoue T, Kiyoto S, et al.: Detection of isolated ipsilateral regional lymph node recurrences by F18-fluorodeoxyglucose positron emission tomography-CT in follow-up of postoperative breast cancer patients. Breast Cancer Res Treat 2011;130:267-272.

$\checkmark 12$ Moossdorff M, Vugts G, Maaskant-Braat AJ, et al.: Contralateral lymph node recurrence in breast cancer: Regional event rather than distant metastatic disease. A systematic review of the literature. Eur J Surg Oncol 2015;41:1128-1136.

13 van Wely BJ, Teerenstra S, Schinagl DA, et al.: Systematic review of the effect of external beam radiation therapy to the breast on axillary recurrence after negative sentinel lymph node biopsy. Br J Surg 2011;98: 326-333.
Kaur P, Kiluk JV, Meade T, et al.: Sentinel lymph node biopsy in patients with previous ipsilateral complete axillary lymph node dissection. Ann Surg Oncol 2011; 18:727-732.

15 Fan Y, Xu B, Liao Y, et al.: A retrospective study of metachronous and synchronous ipsilateral supraclavicular lymph node metastases in breast cancer patients. Breast 2010;19:365-369.

16 Braunstein LZ, Niemierko A, Shenouda MN, et al.: Outcome following local-regional recurrence in women with early-stage breast cancer: Impact of biologic subtype. Breast J 2015;21:161-167.

17 Pedersen AN, Møller S, Steffensen KD, et al.: Supraclavicular recurrence after early breast cancer: A curable condition? Breast Cancer Res Treat 2011;125:815-822. 18 Moossdorff M, van Roozendaal LM, Strobbe LJ, et al.: Maastricht Delphi consensus on event definitions for classification of recurrence in breast cancer research. J Natl Cancer Inst 2014;106:pii:dju288.
19 Aebi, S., Gelber S, Anderson SJ, et al.: Chemotherapy for isolated locoregional recurrence of breast cancer (CALOR): A randomised trial. Lancet Oncol 2014;15: 156-163.

20 Interdisziplinäre S3-Leitlinie für die Früherkennung Diagnostik, Therapie und Nachsorge des Mammakarzinoms. Version 4.0 2017 https://www.leitlinienprogramm-onkologie.de/fileadmin/user_upload/LL_ Mammakarzinom Langversion_4.0.pdf (cited 2017).

1 Loblaw DA, Prestrud AA, Somerfield MR, et al.: American Society of Clinical Oncology Clinical Practice Guidelines: Formal systematic review-based consensus methodology. J Clin Oncol 2012;30:3136-3140. 